\title{
GESTION DE LA DOULEUR AIGUË ET CHRONIQUE EN EXPÉRIMENTATION ANIMALE
}

\section{ACUTE AND CHRONIC PAIN MANAGEMENT IN ANIMALS USED IN EXPERIMENTAL PROCEDURES}

Par Patrick VERWAERDE

(Communication présentée le 25 mars 2021, note acceptée le 7 octobre 2021)

Mots-clés : nociception, allodynie, hyperalgésie, chronicisation, analgésie précoce, analgésie multimodale, analgésiques non conventionnels

Keywords: nociception, allodynia, hyperalgesia, chronicization, early analgesia, multimodal analgesia, unconventional analgesics

\section{INTRODUCTION}

La prise en charge de la douleur animale et le respect de la règle des 3R (Remplacer, Réduire, Raffiner) constituent l'un des enjeux éthiques majeurs de l'expérimentation animale moderne. Les avancées récentes de l'algologie animale et humaine soulignent néanmoins l'importance méthodologique d'une analgésie adéquate pour prétendre acquérir des données expérimentales interprétables d'un point de vue scientifique. Les douleurs aigues ou chroniques secondaires notamment aux gestes chirurgicaux peuvent en effet être à l'origine de biais pantropes mal reproductibles dans de nombreux domaines de recherche (neurologiques, métaboliques, hémodynamiques, endocriniens, inflammatoires, immunitaires, infectieux, ...). Définie comme "une expérience émotionnelle ou sensorielle aversive qui provoque des réactions motrices et végétatives initialement protectrices, conduisant à des comportements d'évitement pouvant modifier durablement le comportement spécifique de l'individu ", la douleur est une sensation discrimi- nante qui repose sur l'intégration supra-thalamique des influx nerveux nociceptifs issus de la périphérie. En clinique comme en expérimentation animale, il convient cependant de distinguer la douleur (i.e. sensation), de la nociception (i.e. processus infra-thalamique de la sensibilité algique). La nociception chez les vertébrés regroupe l'ensemble des phénomènes nerveux en lien avec la genèse, la transmission et la modulation des influx nerveux nociceptifs atteignant le thalamus par les voies spinothalamiques. Elle se traduit par diverses réactions neuro-végétatives, neuroendocriniennes et motrices. Au-delà d'être l'une des causes de la morbimortalité periopératoire (Anand et al. 1992), la nociception, par ses conséquences à court et moyen termes, introduit dans la majorité des études utilisant des animaux des biais de sélection, d'information, de confusion à l'origine de biais cognitifs non maitrisables sans une analgésie stricte durant notamment les phases pré-, per- et postopératoires (Sanoja et al. 2005).

1 : Pr Patrick VERWAERDE, DMV, MSc, PhD, HDR, Dipl ECVECC, Chef du pôle Anesthésie-Réanimation-Urgence-Soins intensifs de I'Hôpital Universitaire Vétérinaire d'Alfort, Ecole Nationale Vétérinaire d'Alfort, Président de la Commission Nationale de Protection des Animaux Utilisés à des Fins Scientifiques (CNPAuFiS) patrick.verwaerde@vet-alfort.fr 


\section{NOCICEPTION, DOULEURS ET CHRONICISATION}

Comme toutes effractions cutanées, une intervention chirurgicale aussi minime soit-elle est à l'origine d'une stimulation de « récepteurs à la douleur » appelés aussi nocicepteurs. L'activation de ces nocicepteurs uni ou polymodaux génère des influx nerveux qui par une série de neurones nociceptifs, convergent vers la corne dorsale de la moelle épinière où ils subiront différentes modulations (inhibitrices et/ou amplificatrices). À la suite, ces influx nociceptifs remontent vers le thalamus où ils seront responsables des effets neurovégétatifs (tachycardie, tachypnée, poussées hypertensives, ...) et neuroendocrines (libération d'adrénaline, majoration $\mathrm{ACTH} /$ cortisol, ...) caractérisant la nociception. Chez l'animal en vigilance normale, l'intégration corticale de ces informations nociceptives est à l'origine de sensations discriminantes appelées douleurs (Figure 1).

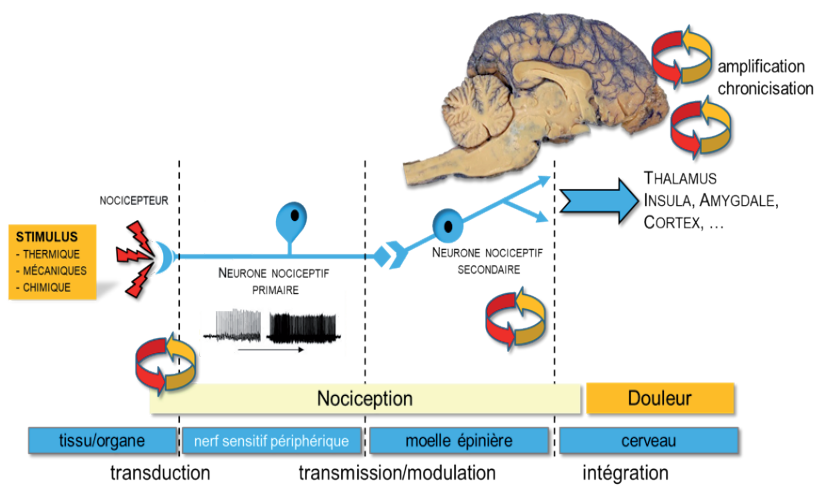

Figure 1 : Schéma illustrant les voies de la nociception et de la douleur, ainsi que les principales boucles d'amplification/de chronicisation de la douleur aiguë periopératoire.

D'un point de vue clinique mais aussi pathogénique, il est usuel, tant chez l'homme que l'animal, de distinguer les douleurs aigueës, des douleurs chroniques. Si les premières se caractérisent par un excès de nociception en lien direct avec les lésions/traumatismes/stimulations algiques initiatrices, les douleurs chroniques apparaissent plus complexes et sans lien, autre qu'historique, avec la stimulation de nocicepteurs. Bien qu'encore mal élucidées, certaines douleurs chroniques constitueraient un continuum pathogénique avec les douleurs aiguës postopératoires. Des avancées en matière d'algologie ont permis depuis quelques années d'identifier divers facteurs pré-, per- et postopératoires favorisant la chronicisation de la douleur aiguë periopératoire (Chapman \& Vierck, 2017). Sans être exclusifs, divers facteurs de chronicisation identifiés sont à rapprocher des phénomènes neuropathiques d'hyperalgésie périphérique et/ou médullaire/centraux responsables respectivement d'une allodynie (sensation douloureuse à la suite d'une stimulation normalement non algogène) et d'une hyperalgésie (sensation douloureuse plus intense que normale à la suite d'une stimulation faiblement algogène). Si ces mécanismes d'amplification de la nociception aiguë sont initialement à support surtout biochimique (inflammation locale, stimulation des récepteurs AMPA/NMDA, ...), leur prolongation/répétition ou plus largement leur non-prise en charge thérapeutique peut être à l'origine d'une plasticité neuronale et gliale médullaire et/ou centrale qui explique en partie la dimension neuropathique persistante de certaines douleurs chroniques postopératoires.

\section{PRINCIPES D'UNE PRISE EN CHARGE ANALGÉSIQUE ADAPTÉE}

La gestion des douleurs aiguës et chroniques animales suppose dans le quotidien des études et de leur élaboration, de prendre conscience des réalités scientifiques qui sous-tendent la nociception et la chronicisation algique postopératoire. En outre, la juste prise en charge des douleurs requiert de respecter quelques principes simples.

Dans un premier temps, il est essentiel de ne pas confondre l'existence d'une narcose (abolition de la vigilance et atténuation des sensations) obtenue par des médicaments dits hypnotiques (isoflurane, propofol, alphaxalone, barbituriques, ...) et une analgésie définie par l'interruption du signal nociceptif et de son intégration par des médicaments ayant une action périphérique, médullaire et/ou centrale. Si une narcose adéquate garantit l'absence d'intégration corticale, elle est sans influence significative sur les phénomènes infra-thalamiques de la nociception et ses conséquences neurovégétatives et neuroendocrines. Ainsi, pour toutes procédures chirurgicales, même de courte durée, une analgésie peropératoire adéquate doit s'appuyer au minimum sur des médicaments ou techniques à potentialité analgésique vraie comme les alpha2 agonistes, les mu-agonistes pleins et/ou les anesthésies locorégionales.

Parallèlement, l'identification et la prévention systématique des facteurs susceptibles de favoriser la chronicisation de la douleur opératoire apparaissent comme indispensables pour espérer réduire la morbidité et éviter les biais induits par les douleurs chroniques. Sans être exhaustif (Chapman \& Vierck, 2017), les principaux facteurs de chronicisation identifiés chez l'homme et pouvant être rencontrés en expérimentation animale sont le non-respect des bonnes pratiques anesthésiques et analgésiques periopératoires, les lésions nerveuses par compression ou section, les chirurgies avec ouverture cavitaire (thoracotomie, laparotomie), les douleurs postopératoires sévères, les chirurgies délabrantes, ainsi que les chirurgies de longue durée et/ou pratiquées avec une maitrise technique insuffisante (Tasmuth et al. 1999 ; Maguire et al. 2006). Si chaque facteur de chronicisation réclame une prise en charge préventive adaptée, certains requièrent une approche pharmacologique complémentaire à l'analgésie per- et postopératoire (médicaments co-analgésiques ou analgésiques non conventionnels), quand d'autres supposent une évolution des pratiques notamment zootechniques et des compétences en matière de technique chirurgicale, d'anesthésie et d'analgésie animales.

Il apparait que la maitrise stricte des douleurs aiguës per- et postopératoires constitue la troisième clef de voûte de la gestion des douleurs en expérimentation animale. En expérimentation comme en clinique vétérinaire, l'établissement a priori d'un plan analgésique adapté à l'intensité douloureuse anticipée doit en outre être complété par une approche observationnelle confirmant ou infirmant sa pertinence. La réalité d'une analgésie 
periopératoire suppose de s'assurer systématiquement de l'absence de signes végétatifs traduisant l'existence d'une nociception active ainsi que de l'absence de signes traduisant une douleur, par exemple au moyen d'échelle de grimace telle que validée pour de nombreuses espèces (Langford et al. 2010 ; https://nc3rs.org.uk/grimacescales). Cependant, au-delà de ces deux étapes méthodologiques, pour une analgésie efficace il importe aussi de respecter scrupuleusement 4 règles essentielles de prescription :

- Initier précocement (i.e. dès la période préopératoire), conformément aux travaux de Woolf \& Chong (1993), une analgésie permettant de contrôler la nociception peropératoire ;

- Assurer une analgésie per- et postopératoire multimodale associant des médicaments analgésiques et/ou co-analgésiques (Ciuffreda et al. 2014) ayant des cibles pharmacologiques distinctes sur les voies de la douleur (figure 1) ;

- Adapter selon le principe des paliers analgésiques de l'organisa- tion mondiale de la santé (OMS ; Vargas-Schaffer, 2010), les prescriptions pré-, per et postopératoires en analgésiques et adjuvants anti-hyperalgésiques (anesthésiques locaux, kétamine, amantadine, gabapentinoïdes, antidépresseurs, paracétamol, ...) ; - Respecter les indications et contre-indications des médicaments ainsi que les objectifs de l'étude par une connaissance approfondie et réactualisée de la pharmacologie et la pharmacocinétique des médicaments prescrits.

Au cour de la règle des 3R et de l'éthique en expérimentation animale, la stricte maitrise des phénomènes douloureux potentiellement induits par divers gestes/techniques même mini-invasifs constitue un enjeu scientifique majeur pour acquérir des données biologiques in vivo interprétables. Dans le cadre d'une approche de réciprocité "One Health ", la gestion des douleurs animales et le raffinement des études peut quotidiennement profiter des enseignements issus de la médecine humaine.

\section{REMERCIEMENTS}

Au Docteur Véronique Lassourd-Verwaerde, DVM, MSc, PhD pour la relecture attentive de ce texte.

\section{CONFLITS D'INTÉRÊTS}

Néant

\section{COMITÉ D’ÉTHIQUE}

Néant

\section{BIBLIOGRAPHIE}

- Anand KJ \& Hickey PR. Halothane-morphine compared with high-dose sufentanil for anesthesia and postoperative analgesia in neonatal cardiac surgery. $\mathrm{N}$ Engl J Med. 1992; 326(1):1-9.

- Chapman CR \& Vierck CJ. The Transition of Acute Postoperative Pain to Chronic Pain: An Integrative Overview of Research on Mechanisms. J Pain. 2017 ; 18(4): 359.e1-359.e38.

- Ciuffreda MC, Tolva V, Casana R, Gnecchi M, Vanoli E, Spazzolini C, et al. Rat experimental model of myocardial ischemia/reperfusion injury: an ethical approach to set up the analgesic management of acute post-surgical pain.
PLoS One. 2014, 22;9(4): e95913

- Langford DJ, Bailey AL, Chanda ML, Clarke SE, Drummond TE, Echols S, et al. Coding of facial expressions of pain in the laboratory mouse. Nat Methods. 2010 Jun; 7(6):447-9. https://nc3rs.org. uk/grimacescales

- Maguire MF, Ravenscroft A, Beggs D, Duffy JP. A questionnaire study investigating the prevalence of the neuropathic component of chronic pain after thoracic surgery. Eur J Cardiothorac Surg. 2006; 29(5):800-5

- Sanoja R \& Cervero F. Estrogen-dependent abdominal hyperalgesia induced by ovariectomy in adult mice: a model of functional abdominal pain Pain. 2005; 118(1-2):243-53.

- Tasmuth T, Blomqvist C, Kalso E. Chronic post-treatment symptoms in patients with breast cancer operated in different surgical units. Eur J Surg Oncol. 1999; 25(1):38-43.

- Vargas-Schaffer G. Is the WHO analgesic ladder still valid? Twenty-four years of experience. Can Fam Physician. 2010; 56(6):514-7, e202-5.

- Woolf CJ \& Chong MS. Preemptive analgesia-treating postoperative pain by preventing the establishment of central sensitization. Anesth Analg. 1993; 77(2): 362-79 